https://helda.helsinki.fi

Stronger response of farmland birds than farmers to climate change leads to the emergence of an ecological trap

\title{
Santangeli, Andrea
}

2018-01

Santangeli , A , Lehikoinen , A , Bock , A , Peltonen-Sainio , P , Jauhiainen , L , Girardello , M \& Valkama , J 2018, ' Stronger response of farmland birds than farmers to climate change leads to the emergence of an ecological trap ' , Biological Conservation , vol. 217 , pp. 166-172 . https://doi.org/10.1016/j.biocon.2017.11.002

http://hdl.handle.net/10138/321411

https://doi.org/10.1016/j.biocon.2017.11.002

cc_by_nc_nd

acceptedVersion

Downloaded from Helda, University of Helsinki institutional repository.

This is an electronic reprint of the original article.

This reprint may differ from the original in pagination and typographic detail.

Please cite the original version. 
Santangeli, A., A. Lehikoinen, A. Bock, P. Peltonen-Sainio, L. Jauhiainen, M. Girardello, and J. Valkama. 2018. Stronger response of farmland birds than farmers to climate change leads to the emergence of an ecological trap. Biological Conservation 217:166-172.

Andrea Santangelia Aleksi Lehikoinen ${ }^{\text {a }}$ Anna Bock ${ }^{\text {bcd }}$ Pirjo Peltonen-Sainioe Lauri Jauhiainen ${ }^{\dagger}$ Marco Girardellog Jari Valkamah

a

The Helsinki Lab of Ornithology, Finnish Museum of Natural History, University of Helsinki, Finland

b

Technische Universität München, Hans-Carl-von-Carlowitz-Platz 2, 85354 Freising, Germany

C

Institute for Advanced Study, Technische Universität München, Lichtenbergstrasse 2a, 85748 Garching, Germany

d

Bavarian Environment Agency, Basics of River Engineering, Hydro-Morphology, Hydraulic, Bürgermeister-Ulrich-Straße 160, 86179 Augsburg, Germany

e

Natural Resources Institute Finland (Luke), Management and Production of Renewable Resources, Latokartanonkaari 9, FI-00790 Helsinki, Finland

Natural Resources Institute Finland (Luke), Management and Production of Renewable Resources, FI-31600, Jokioinen, Finland

9

Azorean Biodiversity Group (cE3c) Universidade dos Açores, Dep. de Ciências Agrárias, Rua Capitão João d'Ávila, sn. Pico da Urze, 9700-042 Angra do Heroísmo, Portugal

$\mathrm{h}$

Finnish Museum of Natural History Luomus, P.O. Box 17, FI-00014, University of Helsinki, Finland

DOI: https://doi.org/10.1016/j.biocon.2017.11.002 


\section{Abstract}

Climate change is triggering adaptation by people and wildlife. The speed and magnitude of these responses may disrupt ecological equilibria and potentially cause further biodiversity losses, but this has rarely been studied. Species inhabiting human-dominated landscapes may be particularly negatively affected by human adaptations to climate change. This could be, for example, the case of ground-nesting farmland birds, a group of highly vulnerable species that may be impacted by shifts in the timing of mechanical farming operations in response to climate change. Here we aim to explore whether trends in phenology of breeding ground-nesting birds differ from those of farming practices, and whether differences lead to the emergence of phenological mistiming with detrimental consequences to the birds. To achieve our objective, we ran linear mixed effects models using a 38-year dataset on onset of farming practices (i.e. sowing dates) and laying date of two endangered ground-nesting farmland birds (Northern lapwing and Eurasian curlew) in Finland. We found that timing of farming practices advanced slower than birds nesting phenology, with birds progressively starting nesting before fields are sown. These nests are at high risk of destruction from incoming sowing operations. The results highlight the importance of considering human adaptation responses, in addition to those of wildlife, for implementing species conservation in managed landscapes under climate change.

Keywords

Global climate change; Long-term data; Spring sowing; Breeding phenology; Phenological mismatch; Climate adaptation.

\section{Introduction}

The global climate is warming at such an accelerating rate that climate change is nowadays considered an imminent rather than a future threat to biodiversity and human societies (IPCC, 2013, Pacifici et al., 2017). Impacts of climate change have now been widely documented across a vast spectrum of ecosystems and ecological processes (Scheffers et al., 2016). These impacts span far beyond the well-established shifts in species abundance, distribution and phenology (Scheffers et al., 2016). The reshuffling of species within communities, as a result of the above mentioned climate-induced effects, has the potential to trigger the emergence of novel community interactions. A major role within these novel community interactions will be played by humans and the way they respond and adapt to changing 
climatic conditions (IPCC, 2013, Watson, 2014). Despite their profound impact on the Earths ecosystems, humans, and their adaptation to climate change, have been largely ignored in most of the literature on climate change effects on the environment (Watson, 2014).

Understanding human responses to climate change is crucial for defining effective mitigation measures necessary to preserve biodiversity (Pacifici et al., 2017, Watson, 2014). Essentially, there is a strong need to expand our research focus from the direct impacts that climate change will have on particular species and also incorporate the impacts of changing human pressures in response to climate change. Such an holistic approach is particularly important for understanding climate change impacts on species that are heavily reliant on manmanaged landscapes, such as farmlands (De Snoo et al., 2013).

In cultivated landscapes, farmers, as well as wildlife, may show adaptation responses to climate change. Farmers may advance spring time practices such as soil preparation and sowing, as well as introduce novel crops for cultivation (Bock et al., 2013, Peltonen-Sainio and Jauhiainen, 2014, Peltonen-Sainio et al., 2015), while birds, for example, may advance the onset of breeding (Both et al., 2004, Kluen et al., 2017). Rates of spring phenological shifts have been documented to vary between species and regions, and according to the trophic level (Both et al., 2009, Thackeray et al., 2010). The differential shift between various components of the food chain may lead to phenological mismatches (e.g. between timing of breeding and timing of peak availability of the main food base; Visser and Both, 2005, Visser et al., 2004), and may result in population declines (Both et al., 2006). The ecological consequences of possible differential phenological shifts driven by climate change have so far been mainly investigated within predator-prey type of systems (Visser and Both, 2005, Visser et al., 2006, Visser et al., 1998). However, species dependent on man-managed landscapes are typically highly vulnerable to anthropogenic activities (Arlettaz et al., 2010, Grüebler et al., 2012, Santangeli et al., 2015). Within these systems, various pressures and factors may trigger differential adaptation responses of wildlife and humans to climate change. This may lead to mistiming, with potentially severe ecological consequences to wildlife. So far, potential phenological mistiming induced by climate change within a human-wildlife system has been largely neglected (but see e.g. Kleijn et al., 2010).

Farmland landscapes represent an ideal opportunity to explore potential phenological mistiming between anthropogenic activities and wildlife under climate change. Farmland biodiversity has a long history of adaptation to man-managed landscapes in Europe (Pain and Pienkowski, 1997), but over the past few decades has been severely impacted by the intensification of agricultural practices (Sanderson et al., 2016, Jiguet et al., 2016). Specifically, the mechanization of agriculture often leads to the destruction of nests of groundnesting species, which are now severely declining (Bas et al., 2009, Grüebler et al., 2012, Pain and Pienkowski, 1997, Santangeli et al., 2014, Santangeli et al., 2015). The degree of this impact may vary, but may be exacerbated due to growing phenological mistiming between bird reproduction and farming practices as a result of climate change. For example, 
the above mentioned mistiming may result in ground-nesting birds starting to lay eggs on yet unsown arable fields in Northern Europe. Consequently, these nests would be at high risk of destruction when the fields are sown. It is thus relevant to simultaneously understand how farmers and birds are responding to climate change in order to foresee potential impacts of climate change and develop effective solutions. This is particularly important at high latitudes where climate is changing most rapidly and, e.g., systematic earlier sowings have already taken place when compared to 1970s and 1980s (Peltonen-Sainio and Jauhiainen, 2014).

In this study we make use of a 38 year (1974-2011) wide-scale dataset of ringing data of two ground-nesting farmland birds in combination with information on timing of farming practices for the same region and period. We examine whether trends in phenology of breeding birds differ from those of farming practices, and if there is a mismatch between these two that could result in detrimental impacts for the two farmland birds which are already threatened in Europe (BirdLife International, 2015). We also explore the impacts of different weather parameters (temperature and precipitation during late winter to spring time) on timing of sowings and phenology of breeding birds. Finally, we discuss the implications of the findings, and how these span far beyond the species and region studied here.

\section{Materials and methods}

\subsection{Study species and study landscape}

We use as study species two farmland birds of conservation concern in the European Union owing to their recent population declines, the lapwing Vanellus vanellus and the curlew Numenius arquata. Both species are threatened by the intensification of agricultural systems in recent decades, particularly the mechanization of practices that increased nest destruction on arable fields. The two species breed across large regions of Finland and are heavily reliant on arable fields for nesting (Fig. S1). Reliance on arable fields has dramatically increased over the past four decades, with over $70 \%$ of curlew nests and $90 \%$ of lapwing nests found on arable land during the period 2000-2011 (see Fig. S1). This is most likely the result of an expansion of the arable areas, which became progressively more available to the two species at the expense of grassland (i.e. within a farmland intensification process), and a reduction in hedgerows.

The two bird species were selected because of their breeding biology and distribution which match our research purpose. Specifically, they are both ground-nesting species that are known to be impacted by mechanical sowing of arable fields in early spring at high latitudes (http://datazone.birdlife.org/home). In northern Europe, mechanical spring sowing broadly coincides with the period when lapwings and curlews start laying eggs (Kluen et al., 2017, Peltonen-Sainio and Jauhiainen, 2014). Consequently, if eggs are laid on yet unsown fields in spring, they are at very high risk of being destroyed by the incoming mechanical sowing. In Finland, a large proportion of lapwing and curlew nests are placed on arable land, including on barley fields. Lapwings and curlews typically make one single breeding attempt per year, 
but replacement clutches are possible when the first nesting attempt fails at an early stage (Grant et al., 1999). Hence, their breeding biology makes these species much more vulnerable to specific farming practices compared to species that breed multiple times during the breeding season. Over the past few decades, populations of lapwing and curlew have been found to progressively advance their onset of breeding as a result of climate change in Finland (Kluen et al., 2017). Similarly, farmers have also adjusted to climate change by advancing farming practices, such as the date of cereal sowing in spring (Peltonen-Sainio and Jauhiainen, 2014). In Finland, $51 \%$ of the utilized agricultural area is used for cereal cultivation, mostly spring barley (22\%) and oat (16\%), whereas fodder grasslands cover $25 \%$ of the total utilized agricultural land, and much less (3\%) is covered by pasture (Niemi and Ahlstedt, 2011). Based on the above, it is interesting and relevant to investigate potential effects of weather parameters in determining the onset of the two species breeding in early spring, as well as the onset of farming practices, such as sowing, which take place in the same period as the birds start breeding and may thus cause nesting failure.

\subsection{Phenological data}

We used nestling ringing data available from 1974 to 2011 across the breeding range of curlew and lapwing within Finland. The data have been collected by volunteer bird ringers with a valid ringing license and following standard protocols for bird handling and ringing. As both species are precocial, nestlings were mainly ringed at or close to their nest at the age of one to two days old. We excluded all ringing data on older chicks because it was not possible to accurately determine their age and, consequently, the hatching and laying date. The database used here is collated and handled by the ringing centre of the Finnish Museum of Natural History. Overall, a total of 1507 ringing data from curlew chicks, and 1493 from lapwing chicks were available from across the 38 years of study (1974-2011). We calculated the day of the year (DOY) for each available observation (hatching date DOY of a clutch), and subtracted from these the number of incubation days of each species in order to derive the DOY value for laying date. In doing so, we used available published information on average incubation period for each of the two species that would be closest to the breeding landscape conditions of our study region; i.e. 27 days incubation period for the lapwing (Larsen et al., 2003), and 29.5 for the curlew (Grant et al., 1999).

In spring, lapwings and curlews approach Finland from a south westerly direction on their migration from the wintering grounds (Saurola et al., 2013). Hence, birds breeding in the south-western regions of the country travel a shorter way, and arrive earlier than birds breeding in more northern areas. To account for this spatial pattern, we identified the center of migration flyway (see Fig. 1) based on available ringing data (Saurola et al., 2013) and defined three circular bands, each of $200 \mathrm{~km}$ width, with center on the migration point (Fig. 1). These three bands, while somewhat artificial, cover the majority of the two species range in Finland and allow a time series to be derived for each band while taking into account spatial patterns in the data. Next, from all the observations available within each band and year, we 
derived an average value of DOY specific to each year and band separately for the lapwing and the curlew. This design resulted in three time series per species, one for each of the three bands. However, these time series were not complete for each band, with 19, zero (i.e. complete series) and 10 missing years for the curlew data, and with four, six and five missing years for the lapwing data (from the SW to the NE bands respectively). Missing years were mostly concentrated in the two outer bands and towards the central period of the time series, when ringing effort was lower than average (see Table S1). We used the derived average DOY per species and by year and band in the following models (see below).
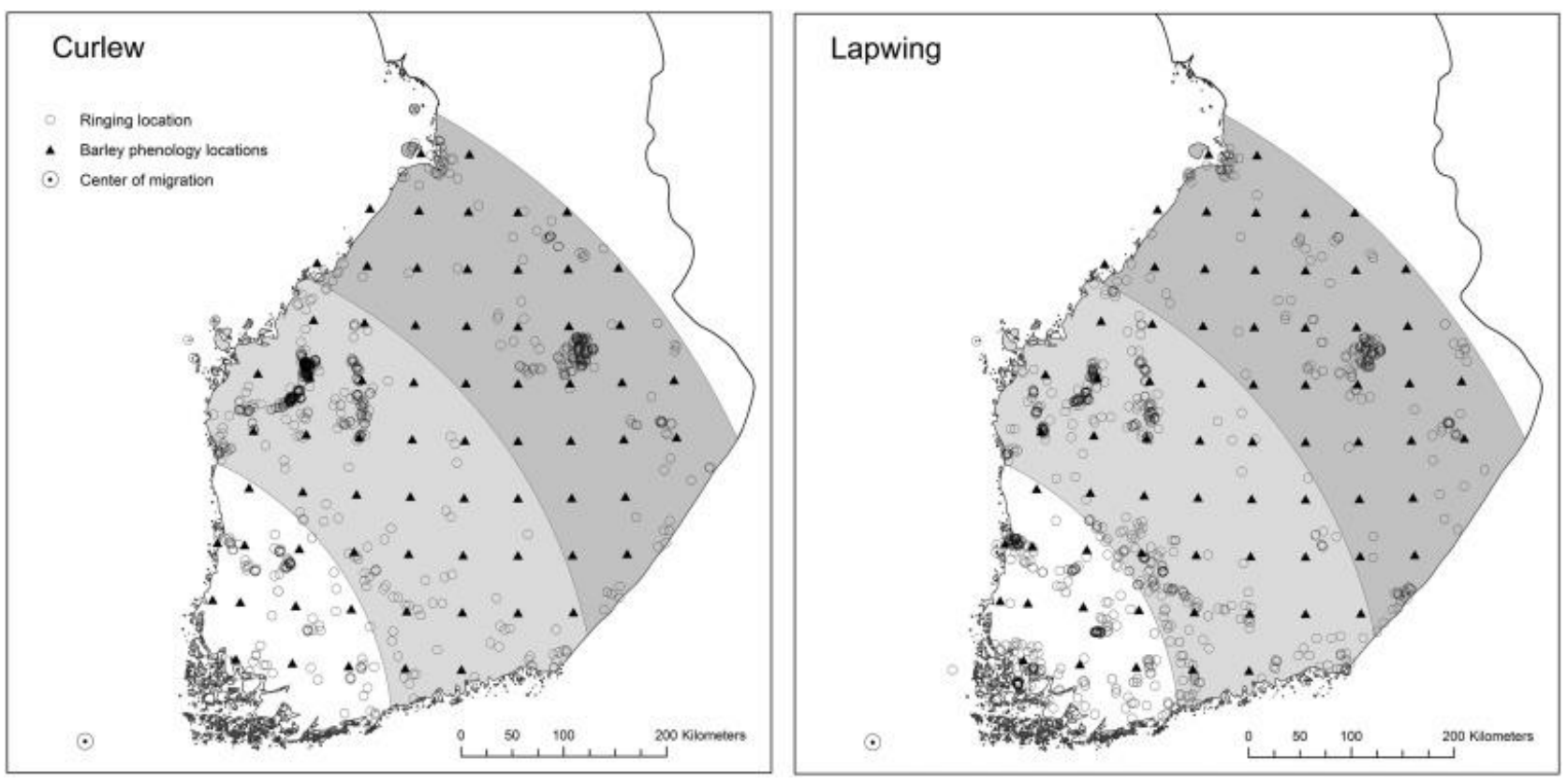

Fig. 1. The distribution of the ringing data (empty circles) for the curlew and the lapwing across south and central Finland (northern Europe). The white dot towards the bottom left of the figure denotes the main migration bottleneck from where the two bird species arrive in spring and that was used as the center point to delineate the three circular bands of $200 \mathrm{~km}$ width (marked in the figures from white to light and dark gray shading). Triangles depict points where the barley sowing phenology was interpolated.

Next, we used modelled sowing dates for spring barley available throughout the same time period and region as the bird data (Fig. 1). Sowing dates were modelled from long term crop trial locations across Finland by considering both environmental and genetic effects (see Peltonen-Sainio and Jauhiainen, 2014 for more details on the crop phenology data). These trials were based on data in which the set of locations varied from year to year. In addition, typical cultivar was included into the trial program for two to five years. Because of the 
imbalance of sample sizes and high variation in the set of cultivars in each trial, the model took into account differences between trial materials. In addition, environmental effects were separated into two components: latitude and distance to coastline of the Baltic Sea. Both have an effect on sowing date, with the magnitude of the effect varying from year to year (Peltonen-Sainio and Jauhiainen, 2014). Here we used sowing date of barley as it is the most common crop across the cultivated areas of Finland. However, sowing dates of other crops, such as oat and wheat, were very similar (within one or 2 days) to those of barley (PeltonenSainio and Jauhiainen, 2014). Similar as for the bird data, we derived a DOY for each sowing date location and then averaged all the sowing DOY within each year and band so as to match the average DOY of the birds time series. These modelled sowing dates closely resemble the real sowing time in Finland (Peltonen-Sainio and Jauhiainen, 2014).

Finally, we obtained weather data from the Finnish Meteorological Institute on monthly temperature and precipitation (months from February to May; interpolated at the $10 \mathrm{~km}$ resolution) covering the same period and spatial extent as the data described above. Again, we derived the average monthly temperature and precipitation for each month from February to May and within each year and band. Weather conditions during this period are the most critical in determining the timing of farming practices (Peltonen-Sainio and Jauhiainen, 2014) and bird phenology (Kluen et al., 2017).

\subsection{Statistical analyses}

We ran three separate Linear Mixed Models (LME), one for the phenology of lapwing, one for the phenology of the curlew, and a third one for the sowing phenology. Each model had the same structure, which included DOY (for lapwing, curlew or sowing within each year and band) as the response. The set of predictors included monthly temperature and precipitation for the months from February to May (eight in total) and year, included as a continuous variable. Band identity (from one to three; see Fig. 1) was integrated as a random effect, to account for pseudo-replication due to multiple observations within each band over the study period (see above). Prior to analyses, we centered all predictors to their mean, to ease comparison of effect size between them. We also checked for collinearity among the predictors, but no issues were identified (i.e. all pairwise correlations $<0.5$ ). No outliers were identified in the dataset. We then used a multimodel inference approach as detailed by Burnham and Anderson (2002). We first computed all possible model combinations using all predictors (see above). Models were ranked using the Akaike Information Criterion (AIC). Next, we selected the $95 \%$ best ranked set of models based on their Akaike weights and performed model averaging on this set. In doing so, the estimated coefficients and standard errors of each predictor were derived from the weighted average of the coefficients across the selected set of models weighted by the relative Akaike weight of the model (using the package MuMin; Bartoń, 2016). We manually checked all the confidence sets of models. These included less than half of all the total model combinations, with most of the models within each set including five predictors. We validated the models by inspecting the residuals 
for unexplained patterns and for temporal autocorrelation using the autocorrelation function, but no residual temporal autocorrelation was detected (see Supporting material Fig. S2). The total sample size available, after excluding years and bands with no data, was 85 sample units (data for each band and year) for the curlew model, 99 for the lapwing model (see also Table S1), and 114 for the sowing phenology model.

Finally, we restructured the database in order to explicitly test for the interaction between trends in timing of sowing of barley with those in phenology of lapwing or curlew. To do so, we combined all phenology data into a single variable (named DOY), and created a categorical variable (arbitrarily named "species", with three classes, i.e. barley, lapwing, curlew) indicating whether the DOY referred to timing of barely sowing, lapwing phenology or curlew phenology in each year and band. Year was also present as a continuous variable. We then ran two separate models, one including only data on barley and lapwing phenology (sample size $=213$ units, i.e. phenology by year, band and "species") and one with only data on barley and curlew phenology (sample size $=199$ ). In each of the two models, DOY was the response variable, and an interaction between "species" (barley vs lapwing or barley vs curlew; as categorical) and year (continuous) was tested. Each of these models had the same random structure as those described above. Analyses were performed in R 3.3.2 (R Core Development Team, 2015).

\section{Results}

We provide evidence that both the birds breeding phenology and the timing of sowings have significantly advanced over the 38 years study period (Fig. 2, Table 1). However, the magnitude of the advance in phenology was different between farming practices and birds. We show that both lapwing and curlew laying dates have advanced five times faster compared to the advance in sowing dates (Fig. 2; statistics for the interaction term between sowing and lapwing phenology: $F_{1,193}=25.7, p<0.001$; and interaction between sowing and curlew phenology: $\left.F_{1,171}=25.8, p<0.001\right)$. The mismatch between bird phenology and sowing time has been growing over the past two decades, with both lapwing and curlew nowadays laying eggs on average 8 days before the sowing date of barley fields in Finland. Furthermore, we show that sowing date of barley is strongly associated with late-winter and early spring weather conditions (Table 1). Low temperatures during late winter and early spring, and high precipitation during May trigger a significant delay in sowing dates of barley (Table 1). Conversely, the association between the two bird species phenology and climatic variables was weak, with only a significant impact of April temperatures on lapwing laying date (Table 1). Specifically, low temperatures in April were associated with a delay in lapwing laying date. 


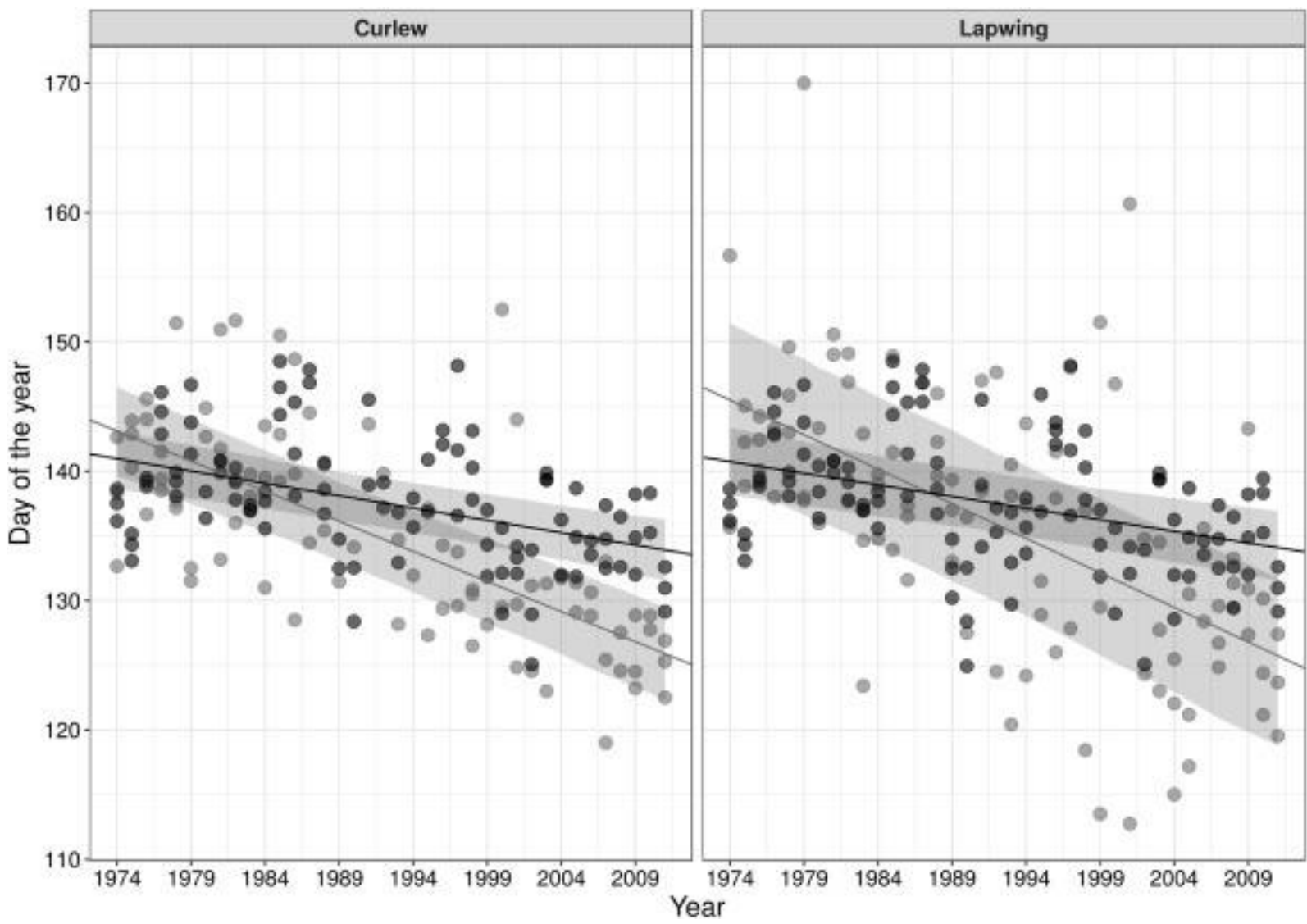

Barley
Birds

Fig. 2. Phenological trends (day of the year, $1=1 \mathrm{st}$ January) of sowing dates for barley (dark gray dots and lines in both panels), and laying date (light gray dots and lines) for the curlew and the lapwing (left and right panel). In each panel dots represent the raw bird and barley phenological data. Lines represent fitted linear regression models of laying date or barley sowing, with year as predictor. The $95 \%$ confidence intervals are also shown by the gray areas. The results are averages across all three bands shown in Fig. 1. 
Table 1. Full model averaged results showing the effect of the climatic variables and year on the onset of barley sowing date as well as laying date of curlew and lapwing across south and central Finland over the period 1974-2011. Results show the coefficients of the fixed effects, along with the $p$-value and relative importance, of each predictor. The response was expressed in day of the year (DOY), and year was included as continuous variable aimed at depicting time trends in DOY over the study period. $\mathrm{P}$ stands for precipitation and $\mathrm{T}$ for temperature followed by the relative month (Feb, Mar, Apr and May refer to February, March, April and May, respectively).

\begin{tabular}{|c|c|c|c|c|c|c|}
\hline Model & $\begin{array}{l}\text { Variable } \\
\text { name }\end{array}$ & Estimate & SE & $\mathrm{Z}$ value & $P$ value & Rel. importance \\
\hline \multirow[t]{9}{*}{ Barley } & P_May & 1.15 & 0.27 & 4.24 & $<0.001$ & 1.00 \\
\hline & T_Apr & -3.12 & 0.29 & 10.50 & $<0.001$ & 1.00 \\
\hline & T_Feb & -1.02 & 0.35 & 2.90 & 0.004 & 1.00 \\
\hline & T_May & -0.87 & 0.26 & 3.31 & 0.001 & 1.00 \\
\hline & Year & -0.08 & 0.03 & 3.22 & 0.001 & 1.00 \\
\hline & T_Mar & -0.51 & 0.35 & 1.45 & 0.148 & 0.82 \\
\hline & P_Feb & 0.14 & 0.28 & 0.52 & 0.606 & 0.40 \\
\hline & P_Mar & 0.12 & 0.23 & 0.52 & 0.604 & 0.40 \\
\hline & P_Apr & -0.01 & 0.13 & 0.06 & 0.948 & 0.27 \\
\hline \multirow[t]{9}{*}{ Curlew } & Year & -0.46 & 0.05 & 8.46 & $<0.001$ & 1.00 \\
\hline & T_Mar & -0.44 & 0.62 & 0.70 & 0.483 & 0.50 \\
\hline & T_Feb & -0.46 & 0.67 & 0.68 & 0.494 & 0.49 \\
\hline & T_May & -0.39 & 0.61 & 0.64 & 0.523 & 0.47 \\
\hline & P_Apr & 0.27 & 0.51 & 0.53 & 0.597 & 0.41 \\
\hline & P_Mar & 0.20 & 0.48 & 0.41 & 0.685 & 0.34 \\
\hline & P_Feb & -0.08 & 0.46 & 0.17 & 0.869 & 0.29 \\
\hline & P_May & -0.02 & 0.32 & 0.06 & 0.955 & 0.25 \\
\hline & T_Apr & -0.02 & 0.38 & 0.04 & 0.966 & 0.24 \\
\hline \multirow[t]{9}{*}{ Lapwing } & Year & -0.44 & 0.08 & 5.39 & $<0.001$ & 1.00 \\
\hline & T_Apr & -2.24 & 1.11 & 2.00 & 0.046 & 0.93 \\
\hline & P_Mar & 0.63 & 0.84 & 0.75 & 0.455 & 0.53 \\
\hline & T_Mar & -0.52 & 0.84 & 0.62 & 0.536 & 0.45 \\
\hline & T_May & -0.32 & 0.65 & 0.49 & 0.623 & 0.38 \\
\hline & P_May & -0.15 & 0.51 & 0.29 & 0.772 & 0.30 \\
\hline & T_Feb & -0.06 & 0.51 & 0.11 & 0.911 & 0.27 \\
\hline & P_Feb & 0.01 & 0.49 & 0.02 & 0.987 & 0.26 \\
\hline & P_Apr & 0.01 & 0.42 & 0.01 & 0.989 & 0.26 \\
\hline
\end{tabular}




\section{Discussion}

Here we show that timing of barley sowing has advanced less compared to the onset of nesting of lapwings and curlews in Finland. This mismatch, under modern intensive farming regimes, is likely to cause widespread negative impacts to the breeding success of these, as well as other ground-nesting birds (Kleijn et al., 2010). The detrimental consequences of phenological mismatches between farming practices and breeding success have been recently shown for ground-nesting birds of agricultural landscapes, e.g. the Montagu's harrier Circus pygargus (Santangeli et al., 2014) or the black-tailed godwit Limosa limosa (Kleijn et al., 2010). Specifically, we demonstrate that, towards recent decades, an increasing proportion of lapwings and curlews in Finland start laying eggs on fields which are yet to be sown. This means that incoming mechanical sowing in spring will cause the destruction of an increasing proportion of nests in the country. As the phenological gap increases, destruction of nests may occur when incubation is already at a very advanced stage, leaving no time for the adult birds to produce a replacement clutch. We also provide evidence that farmers rely largely on suitable local (i.e. within Finland) climatic conditions (such as temperature and precipitation during late winter and spring) for deciding when to start sowings in Finland. For example, soil frost and repeated rains are known to delay the onset of sowing (PeltonenSainio and Jauhiainen, 2014). Moreover, farm machinery and soil management systems have changed (Kaukoranta and Hakala, 2008), e.g., towards direct drilling (whereby seeds are directly placed to the ground of stubble fields without previous soil preparation), and farm size has increased during recent decades (Peltonen-Sainio et al., 2017). These are all factors that may contribute to a delay in farming operations in spring. Conversely, the link to local climatic conditions was weaker for the birds. This is most likely due to the fact that these birds are known to adjust the timing of migration largely as a response of broad weather conditions across their wintering range, rather than local weather on the breeding grounds (Rainio et al., 2006, Vähätalo et al., 2004). The fact that temperature in April had a stronger impact on the phenology of lapwing as compared to the curlew may relate to the fact that, in this study system, lapwings may winter closer to their Finnish breeding grounds than curlews (http://datazone.birdlife.org/home). Therefore, at the onset of their breeding season, lapwings may be more directly exposed to the climatic conditions present on their breeding grounds, and consequently more ready to adjust to them, as compared to curlews.

We acknowledge that the data used here may have some limitations, such as the fact that early failed nests (e.g. during the incubation period) are not included in our ringing database. However, we are convinced that this does not represent a relevant bias for this study. If the yearly proportion of nests that failed has remained constant over the studied period, then this would have had no impact on the phenological trends reported here. However, it is possible that the proportion of failed nests may have increased towards more recent years, e.g. as a result of increased phenological mismatch between birds laying date and sowing operations. 
This could have been further exacerbated by the growing reliance of the two species on arable fields over the past decades (see Fig. S1). If this was the case, it would mean that a larger number of early nests during recent years would be missing from our ringing database, and late nests from replacement clutches would be overly represented. Including those early nests in our analyses would have only made the patterns of advance in lapwing and curlew phenology even stronger (as these observations would push the slope of the light gray lines in Fig. 2 even more down towards the recent years), eventually strengthening the already clear patterns reported here. While we do not have detailed long term information on nest failure occurrences and on the causes of them, we can confidently assert that the reported phenological mistiming between the birds breeding and sowing operations will cause nest destruction. This is because in Finland there are no nationally or regionally organized conservation programmes aimed at protecting nests of ground-nesting birds, as it may be the case for e.g. France or Spain (Santangeli et al., 2014, Santangeli et al., 2015). Thus, most of the nests of lapwing and curlew in Finland are left unprotected. These nests are very well concealed on the ground and very difficult to detect by a farmers during sowing operations. Therefore, although we do not have data to demonstrate it, it is most likely that nests placed on yet unsown fields will be destroyed.

A large share of scientific effort largely focuses on predicting impacts of future climate change, or understanding past impacts in terms of shifts in distribution, abundance and phenology (Pacifici et al., 2017, Scheffers et al., 2016). The latter has been typically related to emerging mistiming between components of the food chain (Visser et al., 2004). However, in order to design and implement effective and long lasting conservation strategies for biodiversity, it is crucial that humans, their adaptation responses and consequent impacts, are explicitly considered. A recent study found that the threat that climate change poses on birds and mammals is greatly underappreciated (Pacifici et al., 2017). Here we provide evidence of a largely overlooked threat resulting from the synergistic impact of climate driven mistiming between avian reproduction and human activities in a man-managed landscape. The implications of these results span far beyond the study system considered here. The emergence of mistiming, and possible ecological traps, as a result of differential shifts of human activities and species life-history events in response to climate change could be widespread. Among others, climate change may cause shifts in timing of land-management practices, resource extraction or tourism and recreational activities. All these shifts have potential to trigger the emergence of mistiming between anthropogenic activities and biodiversity, with the latter most likely being negatively impacted. However, opportunities to alleviate the above negative impacts may also emerge as climate will continue to change. For example, at high latitudes farmers may progressively replace spring cereals with autumnsown crops (IPCC, 2013, Peltonen-Sainio et al., 2009). This could lead to win-win adaptation measures for farmers and wildlife, which need to be proactively identified, carefully evaluated and eventually supported.

\subsection{Conservation recommendations}


Our study provides evidence that human responses and adaptations should be more widely investigated in light of their potential to create phenological mismatches with biodiversity under climate change. To aid effective conservation of ground-nesting farmland species under global change, two approaches may be most relevant to conservation practitioners and decision makers. On the one hand, nest protection on cultivated land may help to minimize impacts of nest destruction in the short term. However, this is often costly and laborious (Santangeli et al., 2014, Santangeli et al., 2015). Even when implemented across large regions, e.g. with the aid of conservation citizens, nest protection may lead to a conservation trap, whereby breeding populations are artificially, i.e. trough nest protection, maintained or even increased on costly land-uses, ultimately compromising the long-term financial sustainability of the conservation program (Cardador et al., 2015, Torres-Orozco et al., 2016). Conversely, a bottom-up landscape approach may hold greater potential for improving the conservation status of ground-nesting farmland birds in the long term. To this end, voluntary non-monetary or market-based approaches (such as the Agri-environment scheme measures of the EU) could represent valuable tools to promote long term sustainable farmland practices that would allow the conservation of ground-nesting birds alongside agricultural production (De Snoo et al., 2013, Santangeli et al., 2016, Batáry et al., 2011). In practice, each target measure is most likely to be highly species and context specific (e.g. autumn sowing could be good for lapwing nest survival at high latitudes, but bad for other birds; e.g. Eggers et al., 2011), which strongly highlights the need for gathering evidence on the effectiveness of interventions across a range of conditions. Ultimately, we urge the scientific community to scan and test the effectiveness of approaches and interventions in each specific context and implement actions before the consequences of large scale nest destruction due to emerging mistiming become irreversible.

\section{Acknowledgments}

We are grateful to all bird watchers and ornithologists that participated in the ringing effort over the years, allowing the collection of the enormous amount of data used in this study. We also thank Alberto Pessia for help with organizing the database, and three anonymous referees for providing constructive comments on an earlier draft. AL and AS were funded by the Academy of Finland (grants 275606 and 283664, respectively).

References:

R. Arlettaz, M. Schaub, J. Fournier, T.S. Reichlin, A. Sierro, J.E.M. Watson, V.

BraunischFrom publications to public actions: when conservation biologists bridge the gap between research and implementation. Bioscience, 60 (2010), pp. 835-842

K. BartońMuMIn: multi-model inference R package version 1.15.6 (2016) 
Y. Bas, M. Renard, F. JiguetNesting strategy predicts farmland bird response to agricultural intensity Agric. Ecosyst. Environ., 134 (2009), pp. 143-147

P. Batáry, A. Báldi, D. Kleijn, T. TscharntkeLandscape-moderated biodiversity effects of agrienvironmental management: a meta-analysis Proceedings of the Royal Society BBiological Sciences, 278 (2011), pp. 1894-1902

Birdlife InternationalEuropean Red List of Birds Office for Official Publications of the European Communities, Luxemburg (2015)

A. Bock, T.H. Sparks, N. Estrella, A. MenzelChanges in the timing of hay cutting in Germany do not keep pace with climate warming Glob. Chang. Biol., 19 (2013), pp. 3123-3132

C. Both, A.V. Artemyev, B. Blaauw, et al.Large-scale geographical variation confirms that climate change causes birds to lay earlier Proceedings of the Royal Society BBiological Sciences, 271 (2004), pp. 1657-1662

C. Both, S. Bouwhuis, C.M. Lessells, M.E. VisserClimate change and population declines in a long-distance migratory bird Nature, 441 (2006), pp. 81-83

C. Both, M. Van Asch, R.G. Bijlsma, A.B. Van Den Burg, M.E. VisserClimate change and unequal phenological changes across four trophic levels: constraints or adaptations? J. Anim. Ecol., 78 (2009), pp. 73-83

K.P. Burnham, D.R. AndersonModel Selection and Multimodel Inference: A Practical Information-Theoretic Approach Springer, New York (2002)

L. Cardador, L. Brotons, F. Mougeot, D. Giralt, G. Bota, M. Pomarol, B. ArroyoConservation traps and the long-term species persistence in human-dominated systems Conserv. Lett., 8 (6) (2015), pp. 456-462

G.R. De Snoo, I. Herzon, H. Staats, et al.Toward effective nature conservation on farmland: making farmers matter Conserv. Lett., 6 (2013), pp. 66-72

S. Eggers, M. Unell, T. PärtAutumn-sowing of cereals reduces breeding bird numbers in a heterogeneous agricultural landscape Biol. Conserv., 144 (2011), pp. 1137-1144

M.C. Grant, C. Orsman, J. Easton, et al.Breeding success and causes of breeding failure of curlew Numenius arquata in Northern Ireland J. Appl. Ecol., 36 (1999), pp. 59-74

M.U. Grüebler, H. Schuler, P. Horch, R. SpaarThe effectiveness of conservation measures to enhance nest survival in a meadow bird suffering from anthropogenic nest loss Biol. Conserv., 146 (2012), pp. 197-203

T.F. Stocker, D. Qin, G.-K. Plattner, M. Tignor, S.K. Allen, J. Boschung, A. Nauels, Y. Xia, V. Bex, P.M. Midgley (Eds.), Climate Change 2013: The Physical Science Basis. Contribution of Working Group I to the Fifth Assessment Report of the 
Intergovernmental Panel on Climate Change, Cambridge University Press, Cambridge, United Kingdom and New York, NY, USA (2013)

F. Jiguet, R. Arlettaz, H.-G. Bauer, et al.An update of the European breeding population sizes and trends of the Ortolan bunting (Emberiza hortulana) Ornis Fennica, 93 (2016), pp. 186-196

T. Kaukoranta, K. Hakalalmpact of spring warming on sowing times of cereal, potato and sugar beet in Finland Agric. Food Sci., 17 (2008), pp. 165-176

D. Kleijn, H. Schekkerman, W.J. Dimmers, R.J.M. Van Kats, D. Melman, W.A.

TeunissenAdverse effects of agricultural intensification and climate change on breeding habitat quality of black-tailed godwits Limosa I. limosa in the Netherlands Ibis, 152 (2010), pp. 475-486

E. Kluen, R. Nousiainen, A. LehikoinenBreeding phenological response to spring weather conditions in common Finnish birds: resident species respond stronger than migratory species J. Avian Biol., 48 (2017), pp. 611-619

V.A. Larsen, T. Lislevand, I. Byrkjedalls clutch size limited by incubation ability in northern lapwings? J. Anim. Ecol., 72 (2003), pp. 784-792

J. Niemi, J. AhlstedtFinnish Agriculture and Rural Industries 2011 MTT Economic Research, Agrifood Research Finland, Helsinki, Finland (2011)

M. Pacifici, P. Visconti, S.H.M. Butchart, J.E.M. Watson, F.M. Cassola, C. RondininiSpecies' traits influenced their response to recent climate change Nat. Clim. Chang., 7 (2017), pp. 205-208

D.J. Pain, M.W. PienkowskiFarming and Birds in Europe. The Common Agricultural Policy and its Implications for Bird Conservation Academic Press, London (1997)

P. Peltonen-Sainio, L. JauhiainenLessons from the past in weather variability: sowing to ripening dynamics and yield penalties for northern agriculture from 1970 to 2012 Reg. Environ. Chang., 14 (2014), pp. 1505-1516

P. Peltonen-Sainio, L. Jauhiainen, K. Hakala, H. OjanenClimate change and prolongation of growing season: changes in regional potential for field crop production in Finland Agric. Food Sci., 18 (2009), pp. 171-190

P. Peltonen-Sainio, A. Rajala, H. Kankanen, K. Hakalalmproving farming systems in northern Europe Victor O. Sadras, D. Calderini (Eds.), Crop Physiology: Applications for Genetic Improvement and Agronomy, Elsevier, Amsterdam (2015), pp. 65-91

P. Peltonen-Sainio, L. Jauhiainen, J. SorvaliDiversity of high-latitude agricultural landscapes and crop rotations: increased, decreased or back and forth? Agric. Syst., 154 (2017), pp. 25-33 
R Core Development TeamR: A Language and Environment for Statistical Computing. Version 3.3.2 R Foundation for Statistical Computing, Vienna, Austria (2015)

K. Rainio, T. Laaksonen, M. Ahola, A.V. Vähätalo, E. LehikoinenClimatic responses in spring migration of boreal and arctic birds in relation to wintering area and taxonomy J. Avian Biol., 37 (2006), pp. 507-515

F.J. Sanderson, R.G. Pople, C. leronymidou, et al.Assessing the performance of EU nature legislation in protecting target bird species in an era of climate change Conserv. Lett., 9 (2016), pp. 172-180

A. Santangeli, E. Di Minin, B. ArroyoBridging the research implementation gap - identifying cost-effective protection measures for Montagu's harrier nests in Spanish farmlands Biol. Conserv., 177 (2014), pp. 126-133

A. Santangeli, B. Arroyo, A. Millon, V. Bretagnolleldentifying effective actions to guide volunteer-based and nationwide conservation efforts for a ground-nesting farmland bird J. Appl. Ecol., 52 (2015), pp. 1082-1091

A. Santangeli, B. Arroyo, L.V. Dicks, I. Herzon, A.S. Kukkala, W.J. Sutherland, A. MoilanenVoluntary non-monetary approaches for implementing conservation Biol. Conserv., 197 (2016), pp. 209-214

P. Saurola, J. Valkama, W. VelmalaThe Finnish Bird Ringing Atlas Vol. 1, Finnish Museum of Natural History and Finnish Ministry of Environment, Helsinki, Finland (2013)

B.R. Scheffers, L. De Meester, T.C.L. Bridge, et al.The broad footprint of climate change from genes to biomes to people Science, 354 (2016)

S.J. Thackeray, T.H. Sparks, M. Frederiksen, et al.Trophic level asynchrony in rates of phenological change for marine, freshwater and terrestrial environments Glob. Chang. Biol., 16 (2010), pp. 3304-3313

D. Torres-Orozco, B. Arroyo, M. Pomarol, A. SantangeliFrom a conservation trap to a conservation solution: lessons from an intensively managed Montagu's harrier population Anim. Conserv., 19 (2016), pp. 436-443

A. Vähätalo, K. Rainio, A. Lehikoinen, E. LehikoinenSpring arrival of birds depends on the North Atlantic oscillation J. Avian Biol., 35 (2004), pp. 210-216

M.E. Visser, C. BothShifts in phenology due to global climate change: the need for a yardstick Proceedings of the Royal Society B-Biological Sciences, 272 (2005), pp. 2561-2569

M.E. Visser, A.J. Van Noordwijk, J.M. Tinbergen, C.M. LessellsWarmer springs lead to mistimed reproduction in great tits (Parus major) Proceedings of the Royal Society BBiological Sciences, 265 (1998), pp. 1867-1870 
M.E. Visser, C. Both, M.M. LambrechtsGlobal climate change leads to mistimed avian reproduction A.P. Moller, W. Fielder, P. Berthold (Eds.), Birds and Climate Change (2004)

M.E. Visser, L.J.M. Holleman, P. GienappShifts in caterpillar biomass phenology due to climate change and its impact on the breeding biology of an insectivorous bird Oecologia, 147 (2006), pp. 164-172

J.E.M. WatsonHuman responses to climate change will seriously impact biodiversity conservation: it's time we start planning for them Conserv. Lett., 7 (2014), pp. 1-2 